\title{
Back to reality: transition techniques from short HMD-based virtual experiences to the physical world
}

\author{
Robin Horst $^{1}$ (D) $\cdot$ Ramtin Naraghi-Taghi-Off ${ }^{1} \cdot$ Linda Rau $^{1} \cdot$ Ralf Dörner $^{1}$
}

Received: 9 March 2020 / Revised: 3 January 2021 / Accepted: 20 July 2021

(C) The Author(s) 2021

\begin{abstract}
Every Virtual Reality (VR) experience has to end at some point. While there already exist concepts to design transitions for users to enter a virtual world, their return from the physical world should be considered, as well, as it is a part of the overall VR experience. We call the latter outro-transitions. In contrast to offboarding of VR experiences, that takes place after taking off VR hardware (e.g., HMDs), outro-transitions are still part of the immersive experience. Such transitions occur more frequently when VR is experienced periodically and for only short times. One example where transition techniques are necessary is in an auditorium where the audience has individual VR headsets available, for example, in a presentation using PowerPoint slides together with brief VR experiences sprinkled between the slides. The audience must put on and take off HMDs frequently every time they switch from common presentation media to VR and back. In a such a one-to-many VR scenario, it is challenging for presenters to explore the process of multiple people coming back from the virtual to the physical world at once. Direct communication may be constrained while VR users are wearing an HMD. Presenters need a tool to indicate them to stop the VR session and switch back to the slide presentation. Virtual visual cues can help presenters or other external entities (e.g., automated/scripted events) to request VR users to end a VR session. Such transitions become part of the overall experience of the audience and thus must be considered. This paper explores visual cues as outro-transitions from a virtual world back to the physical world and their utility to enable presenters to request VR users to end a VR session. We propose and investigate eight transition techniques. We focus on their usage in short consecutive VR experiences and include both established and novel techniques. The transition techniques are evaluated within a user study to draw conclusions on the effects of outro-transitions on the overall experience and presence of participants. We also take into account how long an outro-transition may take and how comfortable our participants perceived the proposed techniques. The study points out that they preferred non-interactive outro-transitions over interactive ones, except for a transition that allowed VR users to communicate with presenters. Furthermore, we explore the presenter-VR user relation within a presentation scenario that uses short VR experiences. The study indicates involving presenters that can stop a VR session was not only negligible but preferred by our participants.
\end{abstract}

Keywords Virtual Reality $\cdot$ Transitions $\cdot$ Presentation slides $\cdot$ Asymmetric virtual environments · Games Engineering · Short VR experiences · Offboarding

Extended author information available on the last page of the article 


\section{Introduction}

It is commonly accepted that transition techniques from the physical to a virtual world can enhance the experience of VR users [25, 27, 35-39, 44], such as distance perception improvements or presence perception. These techniques are considered intro-transitions as they transfer users from their physical to a virtual environment. Outro-transitions, which let VR users return from a virtual to the physical world, are not in the focus of research. Literature indicates that intro-transitions can influence the virtual reality (VR) experience more directly and are more important (e.g., [36]). Furthermore, average VR sessions take a considerable amount of time (e.g., 19.7 min [41] or even 38 min [40]), and VR developers (e.g., Oculus/Facebook [8]) recommend breaks of at least 10 to 15 minutes after such sessions. For example, with HMD-based VR, a user would put on an HMD for one VR session, experience the VR for a while, and then put off the HMD for a while. Therefore, it is reasonable that transition techniques for average VR sessions may take longer than techniques used with short experiences. After all, we consider VR sessions to be singular and disjoint events, which reduce the impact of outro-transitions on a potential next session. The next session will be several minutes ahead.

But there are relevant use-cases where VR might be experienced periodically and for only short times so that users likewise must put on and take off HMDs frequently and consecutively. Furthermore, there are use-cases in which timely constraints matter. Here, outro-transitions might be able to influence the overall experience of VR users. In such use-cases, even short transition techniques would take an unreasonable amount of time compared to the actual VR session that the transition leads into. One example is VR usage within demos or presentation-like structures [14, 16, 22, 31]. This application also reflects the educational microlearning trend [20] within the e-learning domain, where different media are used within curricular structures and where learners can switch between media at frequent intervals. For example, a presenter gives a presentation to an auditorium. Both common media (e.g., slides, vidoes, images) and VR is used. Short VR experiences are utilized to enhance parts of the presentation. Presenters can use VR to substitute single slides in his deck with small VR applications [15] to make use of the vividness and interactivity [45] at only specific suitable places within the presentations. However, the audience has individual VR headsets available and switches between the virtual experiences and the slides and put on and take off HMDs frequently every time they switch.

Presentations in auditoria are time-critical events with fixed time schedules. In a such a one-to-many VR scenario, it is challenging for presenters to explore the process of multiple people coming back from the virtual to the physical world at once. The question arises: Who triggers the transitions that signal VR users the end of the VR session? In a traditional presentation, presenters decide when to switch from one to the next slide as they are the active participants of presentations. When VR users can decide when to end the VR experience, it will be challenging to stick to the schedule. Furthermore, asymmetric virtual environments constrain the communication between users [3], which can lead to a lack of mutual communication between the VR user and the presenter in our case that would be for such co-located experiences [4].

At this point, virtual visual cues can help presenters or other external entities (e.g., automated/scripted events) to request VR users to end a VR session and return to the presentation at once. However, giving presenters such a tool to overcome organizational challenges also influences the overall experience of users since the cues are part of their virtual world. Furthermore, giving presenters the ability to initiate transitions could be perceived as a loss 
of control for VR users. Similar to the offboarding concept [17] of virtual experiences, that takes place during and after the VR users take off their HMDs, transitions before this step will influence the overall experience of the audience.

In this paper, we make the following contributions:

- We explore transitions from a virtual world back to the physical world. We introduce this concept as outro-transitions and focus on short and frequent usage times of VR, such as the described presentation scenario. We identified 3 established and propose 5 novel techniques. All 8 transition techniques for outro-transitions can signal VR users to end a VR session without the need for further verbal instructions by presenters.

- We introduce a conceptual model for outro-transitions that also serves as taxonomy for VR transitions. We divide the transition concept into three distinct and sequential phases that a transition consists of: initiation, interlude, and exit. The considered outrotransition concepts are classified within the proposed taxonomy.

- The techniques are evaluated within a user study to draw conclusions on the effects of outro-transitions on the overall experience and presence of participants. We investigate how long outro-transitions should take in a presentation use case. Our user study also distinguishes who triggers the transition to investigate the influence of presenteror user-initiated outro-transitions. Based on that study, we give practical advice about which types of transition presenters should use in their applications to request VR users to end the VR session and ensure all return at once.

This paper is organized as follows: The next section discusses related work. In Section 3, we explore outro-transition techniques. In the fourth section, we state our evaluation of the techniques within our user study. Section 5 provides a conclusion and points out directions for future work.

\section{Related work}

In this section, we focus on related work about concepts and studies on transitions and VR and consider work that uses VR as a medium for presentations.

\subsection{Virtual reality transitions}

Pioneering work on VR and transitioning by Slater, Usoh, and Steed [35] proposed several aspects about transitioning users into virtual worlds and the potential positive impact on the subsequent VR experience. Furthermore, they propose two HMD-based transition techniques. The authors could indicate that taking on a virtual HMD showed a positive association with the corresponding VR experience. In contrast, a virtual door was associated with a negative association by their participants. A study focuses on the influence of these transitions on the experienced presence of the participants.

Following work by Steed et al. [36] describes the importance of considering the complete experience with a VR system, ranging from preparation to enter, to retirement from the place of usage. They state lessons learned from demonstrations that they have given with a VR system and conclude that it is relevant for demonstration scenarios to incorporate possible conversations and discussions after or during the experience. The 
authors divide the procedure of a VR experience into five steps. The instruction step (1) incorporates all pre-entry instructions that users should know before interacting with the VR technology, such as a verbal introduction to the hardware. With the entry step (2) they refer to the few seconds where users enter the system, for example, putting on an HMD or walking into the projection space. They clarify that this is an essential step for the overall experience, as it blocks out the real world, and introduces the virtual. Steed et al. specifically emphasize the importance of smooth transitions from the physical to the virtual world. In the boot-strapping step (3) users learn the controls which is a less crucial aspect for the VR experience of users. The main experience follows (4). Here, users are familiar with the controls and explore the VR scene. The exit step (5) is the last step in their process. They state that this step might be of negligible importance for the experience and that people could exit fast. However, the evaluation of this aspect remains future work. Steed et al. also have put this claim into perspective by describing that the importance of the exit might also depend on the use-case. For example, they describe the exit to be more important in the case of an experiment, where a participant has to fill out a questionnaire directly after the VR experience. They already outline that a controlled exit such as a prompt for the audience to leave might be a valuable tool to perform the exit within such scenarios, but it remains to investigate and evaluate such prompts. We argue that the overall experience of our use-case can be influenced similarly to such an experiment. Instead of a questionnaire, other presentation elements such as slides or physical exhibit stations might follow directly.

Work by Steinicke et al. [37-39] and Neves et al. [27] investigated transition techniques for HMD-based and CAVE VR setups, respectively. Regarding HMD-based VR, they explored the impact of transitional environments on the distance perception in immersive VR [37] and the influence of a gradual transition on their participants' presence. They described transitional environments as virtual replicas of the physical environments in which users would put on HMDs. Users find themselves within the virtual replica and see a virtual portal within the room through which they can see the actual virtual world that they transitioning into. They could show that the participants well perceived this portal transition. The authors could indicate that distances estimations of their participants within the virtual environments significantly improved by using transitional environments. Within their second study [39], they used the same transitional environment and showed a significant increase of their subject's sense of presence. These replica experiments were reasoned upon a preceding experiment by Slater et al. [34], where they propose a virtual ante-room. The participants walked through a door within the virtual ante-room in this study, whereas in the studies by Steinicke, $[37,39]$ the participants initiated the transition effect themselves. Pushing a virtual button within the room started a teleporter visualization. Both the user-initiated transition and the given transition indicated a positive influence on the sense of presence.

Recent work by Valkov and Flagge [44] proposed a concept of smooth immersion into HMD-based immersive virtual environments. They define a smooth immersion as a transition that includes at least four components: The real world, virtual replica, and userinitiated smooth transition that increasingly transitions from the replica to the virtual world, which is the last component. Furthermore, they define a smooth transition as a continuous morphing process where objects of the replica successively turn into objects of the virtual world.

Men et al. [25] investigate the impact of four specific transition implementations on the participants' presence. Their transition techniques build on established film-based techniques between different shots, such as a simple cut transition or a fade to black transition. 
Their study indicated that short and basic transitions were more suitable to support the sense of presence than visually complex techniques that they compared them to.

At last, recent work by Hovhannisyan et al. [17] elaborate on the offboarding process of VR experiences. Opposed to onboarding of an application, offboarding refers to the process of users who want to cancel a subscription of a provider or remove their account, for example, from social networks. The authors refer to the offboarding of VR experiences as a post-immersion process. They argue that an immersive VR experience does not end abruptly, but that the experience fades during and some time after the participants have removed the headset. Consequently, the offboarding process as defined by Hovhannisyan, Henson, and Sood begins right after the concept of outro-transitions. However, it can be argued whether offboarding of VR applications can be extended to include outrotransitions. For example, an outro-transition that shows the audience a camera stream of their physical surrounding might also include sensorimotoric aspects and foster the reorientation of them even before taking off the HMD. Besides scientific work, practitioners' VR installations recently address questions on how to design on- and offboarding for VR experiences. For instance, 'The Collider' by Anagram [2] includes such processes. It integrates them within the narrative of their experience, for example, by letting the participants of their multi-user installation conclude the experience together within a communicative exchange about the experiences. Still, such offboarding rituals and bespoke processes take place after the participants took of the HMDs, which is after the point of the experience that we place an outro-transition.

The above-mentioned related work focuses on intro-transitions that were either omnipresent or initiated by the VR users themselves. Outro-transitions are out of the scope and not further regarded, also since their participants did not use VR in short and frequent intervals. Asymmetric presenter-initiated transition techniques are not mentioned. This insight motivates the exploration of externally triggered outro-transitions within our use-case.

\subsection{Presenters and presentations with virtual reality}

Steed et al. [36] proposed to have a virtual presenter in their ante-room. The presenter represented the experimenter that gave the participants instructions during their studies. The experimenter controlled the virtual presenter visualization from a desktop. They could reinforce the users' sense of traversal by making the presenter visibly transition from the physical to the real world.

Klinker et al. [22] propose a pattern-based approach for creating Augmented Reality (AR) applications. The resulting applications are rather small, for example, one lets users rotate a car and another one compares two cars visually. In their use-case, an entire presentation consists of AR as a medium, so that they do not have to switch between AR and other media. The authors specifically investigate the authoring of those small applications derived from recurring scenarios for an automotive design presentation. Schmalstieg et al. [31] propose another AR presentation system. Again, presenters and audiences can communicate naturally. They can see each other using AR as a medium.

Work by Peter et al. [29] investigates asymmetric interaction concepts that include a presenter and the audience during VR demo situations. They propose the VR-guide role, which can steer the VR users' attention to guide them through the demo. VR-guides use desktop PCs to get involved in the virtual environment. The authors provide the guides with five tools to interact with the VR users and the scene. They focus on pointing and 
highlighting metaphors. Transitioning is not among the tools. Following work by Horst and Dörner [15] explores the creation of bite-sized VR applications and their integration in demos. They indicate that a shift of control from VR users to presenters may not affect the overall experience of VR users in a demo setting that utilizes short VR applications.

The work we mentioned about VR presentation scenarios does not include transitions. Furthermore, existing work exploring the presenter-audience relationship in such use-cases does not investigate the impact of outro-transitions as a link between short VR experiences and other media. The influence of the choice of who triggers such transitions remains unexplored, as well.

\section{Outro-transitions}

This section proposes our model and taxonomy for VR transitions, then describes eight outro-transition techniques and classifies them according to the taxonomy.

\subsection{Model and taxonomy}

Outro-transitions aim to give VR users a non-verbal cue to end their VR experience and smoothly guide them to exit the virtual environment. Generally, we divide a VR transitioning process into three distinct phases within our model, that are initiation, interlude, and exit (Fig. 1):

1. Initiation - As the name suggests, initiation is the aspect that triggers the beginning of a transition. Before the initiation, the actual content of the VR scene that the user transitions from takes place. Different user roles of a VR system can perform initiations, and they can be carried out using different interfaces. For example, Steinicke et al. propose

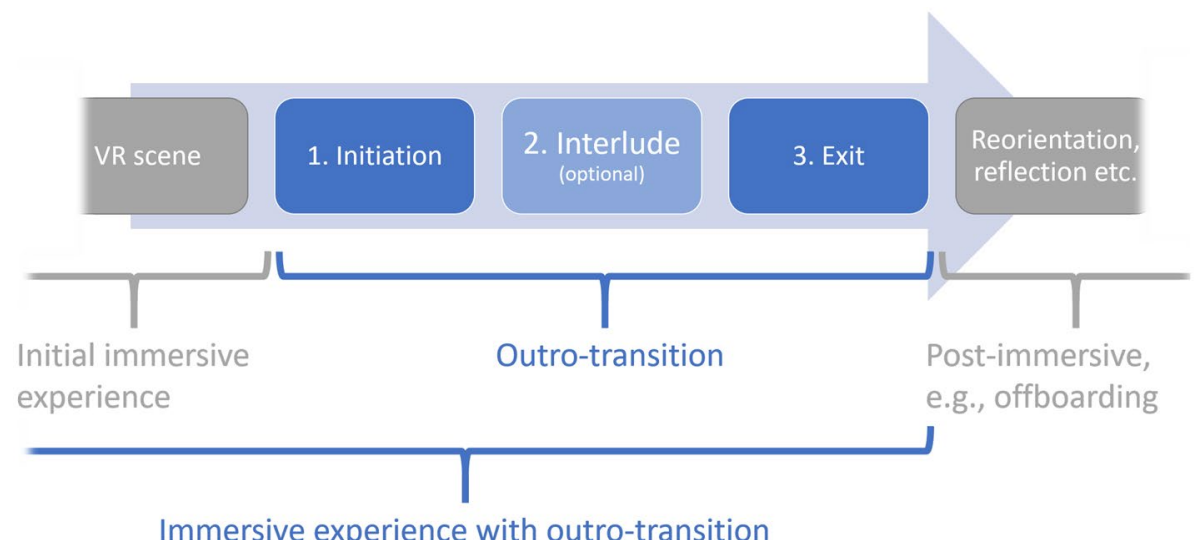

Fig. 1 Our conceptual model of outro-transitions. The three distinct phases also serve as a taxonomy for VR transitions. Outro-transition integrate into the immersive experience of VR users by extending the initial VR scene. After the outro-transition, the post-immersive phase begins, for example, offboarding [17] 
[37] a user-initiated transition by providing a button to the VR users within the virtual room, whereas Slater et al. [34] use an omnipresent transition presented as a simple door within the virtual room. Another possibility is an externally initiated transition. These externally triggered initiations also include presenter-initiations. A presenter could trigger transitions via a desktop interface. External initiations also include automated triggers, where a transition launches without user interaction. Triggers could, for example, be predefined time- or event-based mechanisms.

2. Interlude-While initiation refers to one discrete point in time (e.g., a button click), the interlude phase can take a certain time span. It represents an optional transition state in which VR users find themselves in after transitions are triggered. Here, visualizations and interactions are provided to VR users to steer their attention away from the original VR scene. These can be common transition metaphors and affordances [28], such as teleporters or doors and other mechanisms that connect initiation and exit. The optional interlude allows designers to make transitions less abrupt and prepare VR users for the following exit phase of the immersive experience.

3. Exit-Exits reflect the part of transitions that indicates VR users to end their immersive experience. Examples of HMD-based techniques that point VR users to the end of their experience are fading the view out to black or overlaying instructions on how to take off the HMD. The exit concept includes not only guidance, but also interactions necessary to exit the virtual environment, such as taking off HMDs or stepping outside tracking areas. These last steps of the exit phase are intersecting with the first steps of VR offboarding methodology [17], where it can be argued whether it is part of the immersive experience or already belonging to the post-immersive experience. After the exit, other offboarding methodology can be performed with the participants, such as supporting the VR users' reorientation in the physical surrounding or letting them reflect upon their experiences.

As described, a minimal transition consists only of an initiation and an exit. Such a fast-paced transition guides VR users toward exiting the VR directly after initiating the transition process and without further gradation. Table 1 illustrates how also into-transition examples mentioned within the related work section can be classified and divided using the proposed taxonomy.

\subsection{Transition techniques}

We consider eight transition techniques in this work that can be used as outro-transitions. Figure 2 illustrates them. These transitions were selected because of their short duration and simplicity. Complex and lengthy techniques are not covered in this work, as they would take too much time compared to the relatively short VR experiences we address in our use-case. The techniques are of different types relating to our model and can be classified as interlude-based or exit-based techniques, depending on whether or not a interlude or a exit shapes the major part of the transition. In the following, we describe the techniques in detail, relate them to our taxonomy, and point out further aspects that classify outrotransitions. The following techniques are not determined to a specific initiation and begin at the interlude or the exit state. Possible initiations are mentioned in the previous section. Table 2 illustrates the classification of the proposed transition techniques that follow.

The first technique we consider is the teleporter technique (Fig. 2(a)). This technique builds on visual effects known from science fiction movies such as Star Trek or Star Wars, 


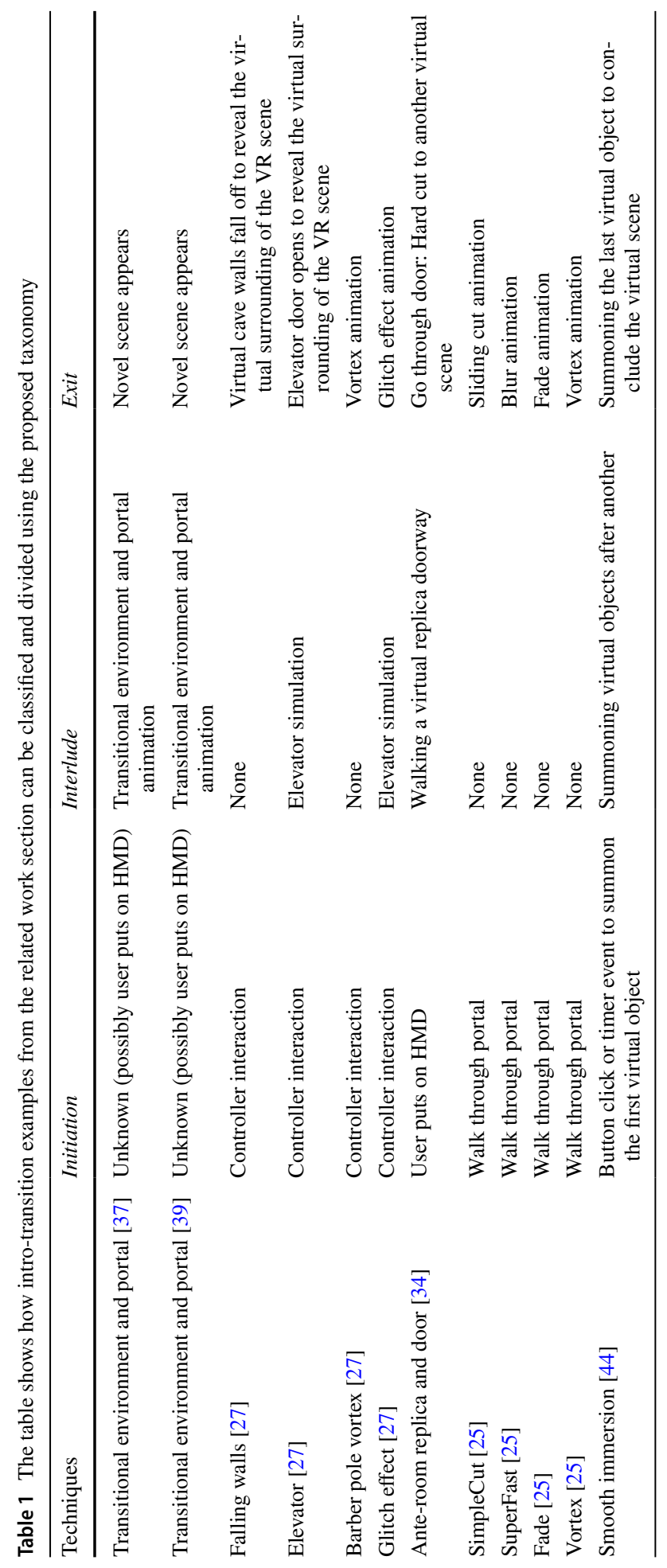




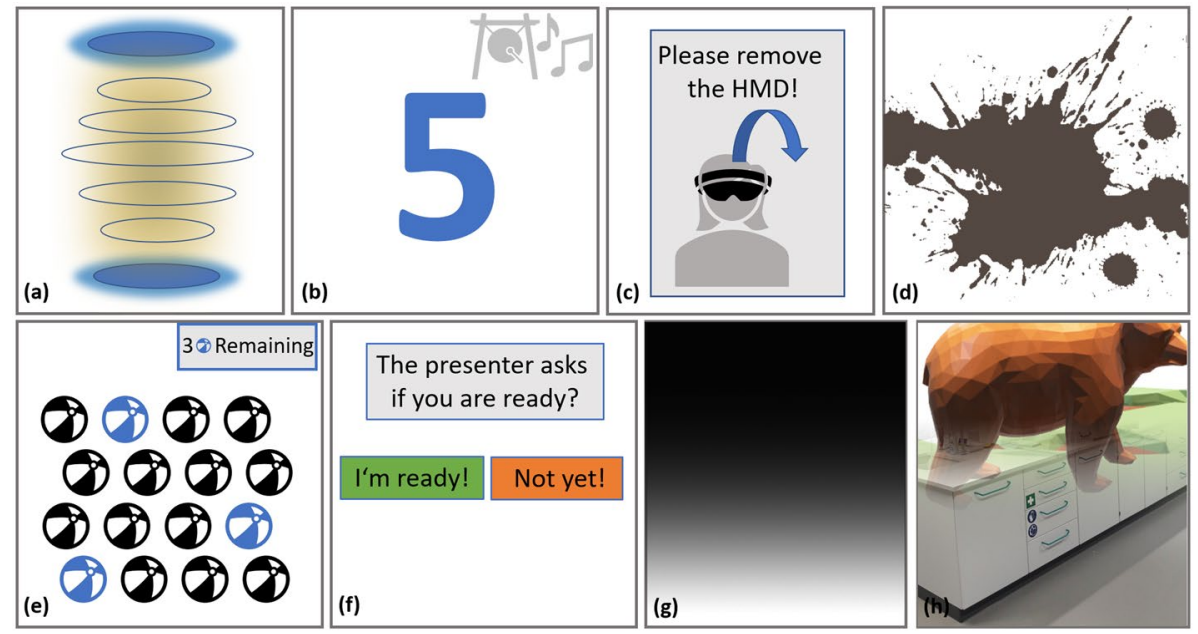

Fig. 2 Illustrations of eight techniques we consider outro-transitions. (a) teleporter, (b) gong, (c) video, (d) wipe, (e) minigame, (f) dialog, (g) fade to black, (h) fade to cam

where teleporters are commonly used to travel between space or time which make teleporters a suitable metaphor for transitions. It is also related to other sci-fi techniques such as portals used in [37-39]. In the interlude state, a teleporter animation processes for a predefined time. After the teleporter animation, an exit must be appended to notify users about the end of the experience, similar to the equivalent for intro-transitions, where the new virtual scene is appended after the animation.

The second transition is gong (Fig. 2(b)). This technique, again, is shaped by its interlude. A visual countdown begins after the transition is triggered. When the countdown reaches 0, a gong audio effect plays. However, gong is a special case. Its exit transports only auditory cues after the countdown. To support gong's exit visually, the technique should incorporate a visual exit, as well. For example, a simple fade effect can conclude it. The design rationale of this technique is related to spiritual methodologies, such as hypnosis or meditation. In these areas, a gong is used to bring practitioners' minds focused on their inward back their physical surrounding. Analogous we propose to transfer this technique to VR as a cue for immersed users to return from the virtual back to the physical world.

Table 2 The table shows the classification of the eight transition techniques that are considered in this work. Four can serve as interlude and four as exit. Only dialog has an influence on the initiation phase

\begin{tabular}{llll}
\hline Techniques & $\begin{array}{l}\text { Serves as } \\
\text { initiation }\end{array}$ & $\begin{array}{l}\text { Serves as } \\
\text { interlude }\end{array}$ & Serves as exit \\
\hline Teleporter & & $\mathrm{X}$ & \\
Gong & $\mathrm{X}$ & $\mathrm{X}$ ) only auditory \\
Video & & $\mathrm{X}$ \\
Wipe & $\mathrm{X}$ & $\mathrm{X}$ \\
Minigame & $\mathrm{X}$ & \\
Dialog & $\mathrm{X}$ & & $\mathrm{X}$ \\
Fade to Black & & & $\mathrm{X}$ \\
Fade to Cam & & & \\
\hline
\end{tabular}


The video technique (Fig. 2(c)) shows a video within the view that displays a video of a person taking off an HMD. It is a less metaphoric cue than the aforementioned techniques and gives the audience direct advice to take off the headsets. Video is a techniques that serves directly as exit. It signals users to take off the HMD without additional exit cues or any supplemental visualizations.

Wipe projects virtual mud within the VR users' field of view after the initiation (Fig. 2(d)). It can be removed using wipe gestures. Behind the mud layers, there is a stream, video, or image of the users' physical environment, which gets more visible the clearer the view gets. Providing cues from the physical world that surrounds the users are used to signal them that the VR experience ended and they are back in their physical surrounding. It can encourage users to take off the HMD when the view was cleared. By using multiple layers of mud, this technique transitions VR users gradually. This design is chosen as a negative incentive or challenge for VR users that they must overcome. At the end, the audience participated actively overcoming the challenge within the transitioning process and is rewarded with a clear view of their surrounding, guiding them positively to the further course of the presentation.

Game and gamification elements find a place in different application areas and can motivate users to interact with a system $[6,7,24]$. Our design rationale for minigame is that game elements can be used to convey tasks that are not intrinsically motivated (e.g., learning tasks for students). When users are immersed and emotionally addressed by a virtual world, our design counteracts the barrier of wanting to stay within the VR instead of exiting at a given point in time that is not self-determined. Minigame gives the audience a positive incentive in the transitioning process to overcome barriers using gamification elements. Furthermore, at the example of a presentation with a larger audience within an auditorium, minigame can also include overarching motivators such as a scoreboard which is available to the entire audience and is adjusted after each VR experience throughout the entire presentation. Minigame functions as interlude, adds game elements to a transition, and provides a short and simple game to be solved by VR users before their exit from the virtual environment (Fig. 2(e)). This minigame shall motivate to exit the VR and create an incentive for frequent usage of VR. Our example technique for a minigame utilizes balls of different colors. Users have to select balls of a given color as fast as possible to obtain a high score at the end. A scoreboard [9] and a timer [6] are used as established gamification elements. After the task is completed, minigame switches to a exit technique to conclude the transition.

The dialog technique is a special case regarding its initiation (Fig. 2(f)). It builds on mutual consent instead of letting one stakeholder (e.g., VR user vs. presenter) decide when to initiate the transition. Both user groups can begin dialog by asking their opposite whether they are ready to exit the VR experience. A visual dialog opens that leaves a binary option to answer positively or negatively and respectively triggers the exit or lets the VR user continue to explore the VR scene. If responded negatively, dialog can be triggered repeatedly by both user-roles later until answered positively. As a fallback, a maximum amount of request can be defined after which the technique continues with the exit. Dialog serves as initiation and interlude within a transition but not as exit. It does not give cues or a prompt for VR users to make them exit the virtual environment.

Finally, we consider two techniques that substitute an interlude and define a transition directly by its exit mechanism. Fade to black (Fig. 2(g)) and fade to cam (Fig. 2(h)) fade the image within an HMD to black or a camera stream that records the physical environment from a first-person perspective. For the latter, small cameras could be attached to the HMDs or cameras used for inside-out tracking that are already present at the technology 
(e.g., [18]) can be used. Both techniques give VR users a cue that the VR experience is coming to an end. Like video, these exit-defined transitions can serve as exit for the mentioned interlude-defined techniques by appending them.

\section{Evaluation}

We evaluated the suitability of the considered transition techniques to be used as outrotransitions within a user study. Furthermore, we evaluated the general concept of outrotransitions within the use-case of short and frequent usage of VR. Our study considers the following six aspects and associated research questions:

[A1] Distinction: How clearly could the participants distinguish between the VR scene's content and the transitions?

[A2] Disturbance: How much did the participants feel disturbed by each transition?

[A3] Duration: How much time may a transition take with regard to the short VR experiences?

[A4] Acceptance: How did the participants accept the concept outro-transitions in general, and how did they rate each transition technique? Were there differences relating to the transitions' hedonic and pragmatic qualities [13]?

[A5] Presence: How did the outro-transitions influence the participants' perceived presence regarding a session that includes several short VR experiences?

[A6] Trigger: How did the aspect of who triggered the transitions impact the presence or other facets of the experience (user-initiated vs. presenter-initiated)?

Next, the evaluation section describes the prototype that we used within our study. Then we describe our user study and the used methodology. Separate sections for analyzing and discussing the results of our study follow.

\subsection{Prototype}

Our techniques were implemented using modern game engine technology. We used Unity [42], an HTC Vive [19], and a gaming PC (GeForce GTX 1070, i7 processor, and 8 GB RAM) to implement and run our prototype implementation. We chose an HTC Vive to be used as VR hardware as it provides front cameras to use for the camera stream of the fade to cam technique. Implementation results are illustrated in Fig. 3. We used a modular system design and encapsulated techniques regarding the three components (initiation, interlude, and exit). This design allows to append each alternative of the categories to each other and create combinations of the techniques. For example, the two fade techniques could serve as exit component for each of the interlude-based transitions. Furthermore, we could easily modify if a VR user- or presenter-initiation of the transitions was required. For the following study, we utilized the established [25-27] fade to black as exit for the techniques that needed one. Table 3 illustrates which combinations were used for our prototype. The presenter-initiation was implemented as a button click on a keyboard and the user-initiation as a button click on the HTC Vive controller. 


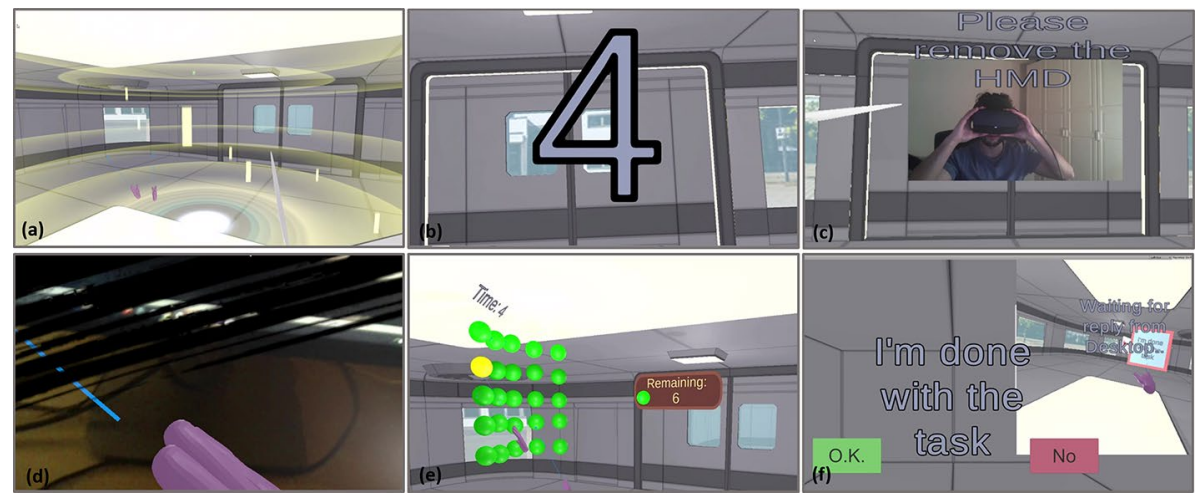

Fig. 3 Screenshot-images that give an impression on possible implementations of the techniques considered in section 3: (a) teleporter, (b) gong, (c) video, (d) wipe, (e) minigame, and (f) dialog. Fade to black and fade to cam are not illustrated

\subsection{User study}

The user study involved 18 unpaid, voluntary participants ( 4 female, aged between 20 and 58 years with $\varnothing 33.28$ and SD 13.56). Their VR experience was captured on a 0-3point scale with $\varnothing 2.33$ and SD 0.57 , where 0 means they never used VR technologies and 3 means they regularly use VR. On that basis, we classify the participants of our study as experienced or expert users in the field of VR.

The study took place as follows: At first, participants were welcomed and then informed about the topic of the study. They were briefly introduced to the user interface of the prototype and the VR hardware. Then the actual task phase of the study started. For this phase, we assigned the participants to groups to evaluate the influence of userinitiation vs. presenter-initiation based on a between-subject design. Still, a withinsubject design was utilized to evaluate the eight techniques, as they were not altered content-wise. We utilized the implementations from our prototype and an existing set of short VR applications, which informed our participants about the use, the structure, and the physical model of a proton-exchange membrane (PEM) fuel cell.

Table 3 The table shows how each phase of the eight transition techniques was implemented within the prototype. The established fade to black served as exit for the techniques that are lacking one or need additional motive

\begin{tabular}{llll}
\hline Techniques & Initiation & Interlude & Exit \\
\hline $\begin{array}{lll}\text { Teleporter } \\
\text { Gong }\end{array}$ & Button click & Teleporter animation & Fade to black \\
Video & Button click & Countdown & Gong sound and fade to black \\
Wipe & Button click & None & Repeating video overlay \\
Minigame & Button click & Mud and active gestures & Cleared camera stream \\
Dialog & Button click & Interactive minigame & Fade to black \\
& Button click to start & Answering dialog request & fade to black \\
Fade to Black & the dialog & & \\
Fade to Cam & Button click & None & Fade to black \\
\hline
\end{tabular}


In the task phase, the experimenter assigned the participants the task to explore the content of the VR applications and to inform themselves about the functionalities of a PEM fuel cell. The VR applications were used in a fixed sequence for all participants as their content built upon each other. However, we used a randomized block design [1] for the order of the transition techniques to encounter a possible learning process' impact or an influence of the content of each application on the scores throughout the study. So, each participant experienced the transition techniques in randomized orders. The participants used each of the VR applications for about 2 minutes and then used a transition before exiting the virtual environment.

Different tools were used within our evaluation to draw conclusions on the research questions concerning A1-A6. Quantitative data was captured with questionnaires filled out in between each of the VR applications. Individual questions were asked to answer questions of the non-standardized aspects A1-A3. Relating to the acceptance (A4), the AttrakDiff $[12,43]$ questionnaire was utilized to measure how the participants perceived the product character [11] of the prototype based on pragmatic qualities (usability) and hedonic qualities [13]. Usability is an established criterion in the evaluation of humancentered products and the assessment of hedonic qualities enables to make assumptions about the educators' desire for pleasure and avoidance of boredom during the usage of outro-transitions.

Each questionnaire incorporated the four following individual questions targeting aspects A1-A3: How clearly could you distinguish the actual VR application from the transition (A1)? Did you know at any time whether a transition was taking place (A1)? How much did you feel disturbed by the transition (A2)? How do you rate the duration of the transition compared to the duration of the VR application (A3)? These questionnaires also contained standardized items from the abbreviated version of the AttrakDiff questionnaire. Our participants used a version of the questionnaire, which was translated into their native language. We used a 7-point semantic differential scale for each of the questionnaire items to capture the scores.

At the end of the user study, after our participants experienced all eight VR applications and transition techniques, they filled out a post-study questionnaire. This questionnaire included the items of the standardized IPQ questionnaire [21] to capture their presence of the entire session (A5). This questionnaire measures the overall presence relating to three established factors [32, 33] - spatial presence, involvement, and experienced reality. This tool enables the evaluation, for example, to draw conclusions on whether our participants' presence over the entire experience was influenced by who triggered the outro-transitions. Again, a translated version was used. Furthermore, our participants were asked to bring each of the techniques in an order from best to worst ('sequence assessment' in the following). This brief post-study questionnaire was also used to capture demographic data and individual comments. A single session of the study was performed within one hour.

\subsection{Analysis of the results}

We aggregated the outcome of the questions for A1 and then performed non-parametric and dependent Friedman tests [10] on the scores for the three aspects A1-A3 to test the data on significant differences. With a threshold for statistical significance of 5\%, all three tests point out that there were significant differences with $p \leq 0.00001$. We conducted further post-hoc tests (after Conover [5]) to identify between which techniques the significant differences occurred. Table 4 shows the output of the Friedman and the post-hoc tests. 
Table 4 also shows the absolute mean values for each of the techniques regarding each aspect, rounded up on four decimal places. We conducted a non-parametric and independent Mann-Whitney U test [23] with a threshold for statistical significance of 5\% on the separated data for each technique (also shown in Table 4) to explore differences between user- and presenter-initiated outro-transitions.

Regarding the perceived separation (A1) of the core VR application and the outro-transition, Table 4 depicts that gong and video have the highest overall scores. Video differs significantly from minigame and wipe, which have the lowest values of our set of techniques, whereas gong also differs from teleporter and dialog. Minigame has the lowest value regarding its separation aspect and differs significantly from every other technique. For the three techniques with the highest overall scores (gong, video, and fade to black), the case distinction about who initiates the transition shows that the initiation-type had no significant influence on their scores. However, the statistical tests confirmed a significant difference between the initiation-type for the other techniques. All were in favor of the presenter-initiated ones. The highest absolute scores were reached by gong and dialog, again, at the presenter-initiated versions. Except for minigame, which has a lower value, all presenter-initiated techniques scored a value of approximately 5 or higher. The only user-initiated technique that has a value of 5 or higher is video. It is the only technique with a higher user-initiated score than the presenter-initiated equivalent. Figure 4 (Q1 and Q2) depicts the distinguished absolute scores for A1.

The second aspect illustrated in Table 4 (disturbance, A2) shows the highest values for fade to black, video, and dialog, ranging from 4.55 to 4.1, respectively. The tests depict that dialog only differs significantly from wipe, whereas fade to black and video additionally differ from fade to cam, and minigame. Wipe received the lowest overall score and differs significantly from every other technique. A significant difference between user- and presenter-initiation is only observed for gong, which also received the highest score (5.4) of all techniques in this category. The next highest values above 5 were received for video (user-initiation), and fade to black (presenter-initiation). The user-initiated gong only has a score of 2, which is the third-lowest value. The next lowest values were both received for wipe with 1.8 (user-initiation) and 0.7 (presenter-initiation). Distinguished absolute scores for A2 are depicted in Fig. 4 (Q3).

The duration of each was assessed as well (Table 4) within our study. The durations for gong, teleport, fade to cam, and fade to black were predefined, whereas the mean duration for video, wipe, minigame and dialog were measured with the following times: gong: $8 \mathrm{~s}$ ( $5 \mathrm{~s}$ countdown $+3 \mathrm{~s}$ sound); teleport: $15 \mathrm{~s}$ ( $8 \mathrm{~s}$ until ring animations appeared $+13 \mathrm{~s}$ until fade was triggered); fade to cam and fade to cam: $2 \mathrm{~s}$; video: approx. $2 \mathrm{~s}$; wipe: approx. $5 \mathrm{~s}$; minigame: approx. 10s; dialog: approx. 11s. The techniques fade to black, dialog, video and fade to cam received a value close to the optimum value of 3 . In contrast, wipe, and teleporter have values below 2 , being the techniques the farthest away from 3 . Only two techniques were rated with a value above 3 (fade to cam and video). The video score differs significantly from every other technique, while dialog and fade to black differ significantly from every technique, except from fade to cam and each other. The consideration of the divided categories after user- and presenter-initiation shows no significant differences between these two cases within each technique. Distinguished absolute scores for A3 are depicted in Fig. 4 (Q4).

We analyzed the outcomes of the AttrakDiff questionnaire and compared the techniques relating again for being user-initiated or presenter-initiated. Figure 5 illustrates each technique relating to their hedonic and pragmatic characteristics. One similarity between both peculiarities is that teleporter and video are arranged closest to the 'desired' region. Teleporter has a higher amplitude towards self-orientation in both graphics, whereas video 


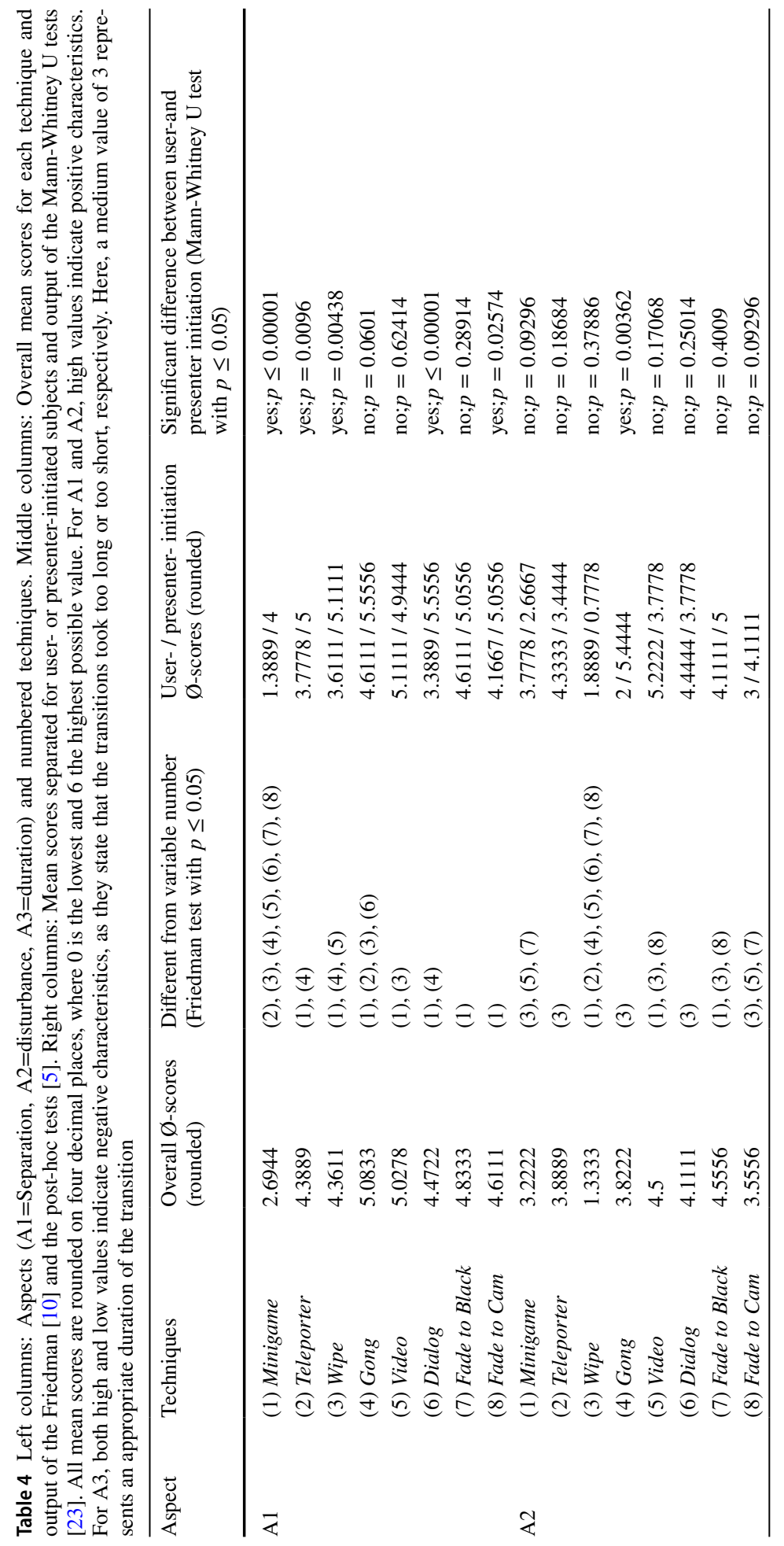




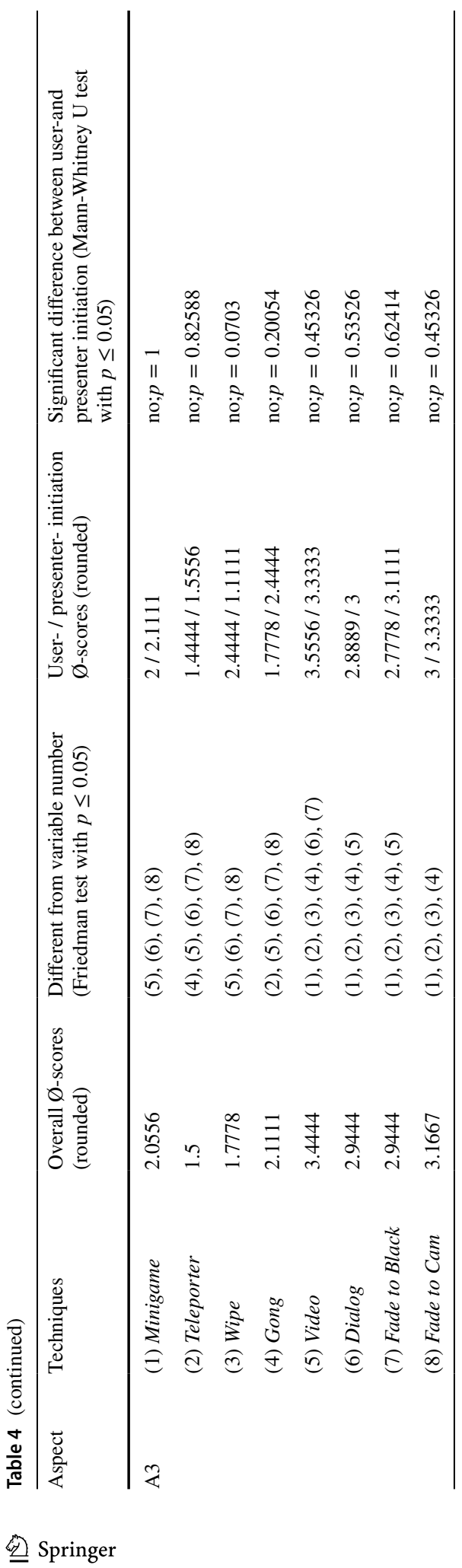


Q1: How clearly could you distinguish

the actual VR App from the transition?

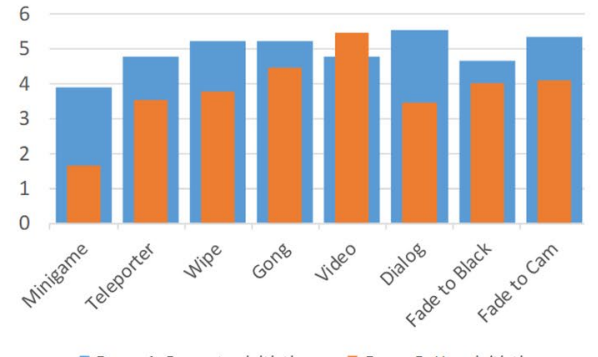

m Group A: Presenter-initiation = Group B: User-initiation

Q3: How much did you feel disturbed by the transition?

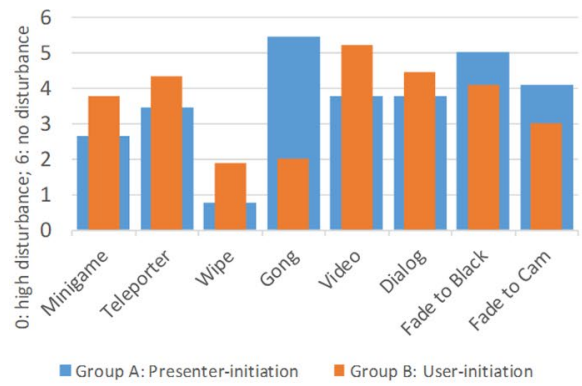

Q2: Did you know at any time whether a transition was taking place?

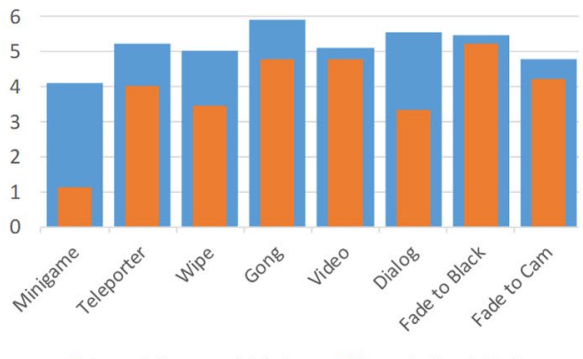

m Group A: Presenter-initiation @ Group B: User-initiation

Q4: How do you rate the duration of the transition with respect to the duration of the VR App?

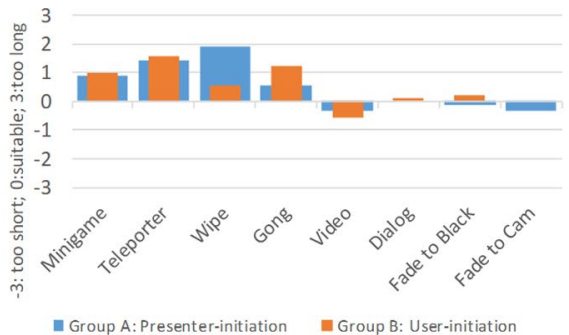

Fig. 4 Bar charts comparing presenter-initiated transitions (blue) with user-initiated transitions (orange). Q1 and Q2 were used to capture A1 (separation), Q3 reflects A2 (disturbance), and Q4 displays the perception of an adequate time for the transitions (A3)

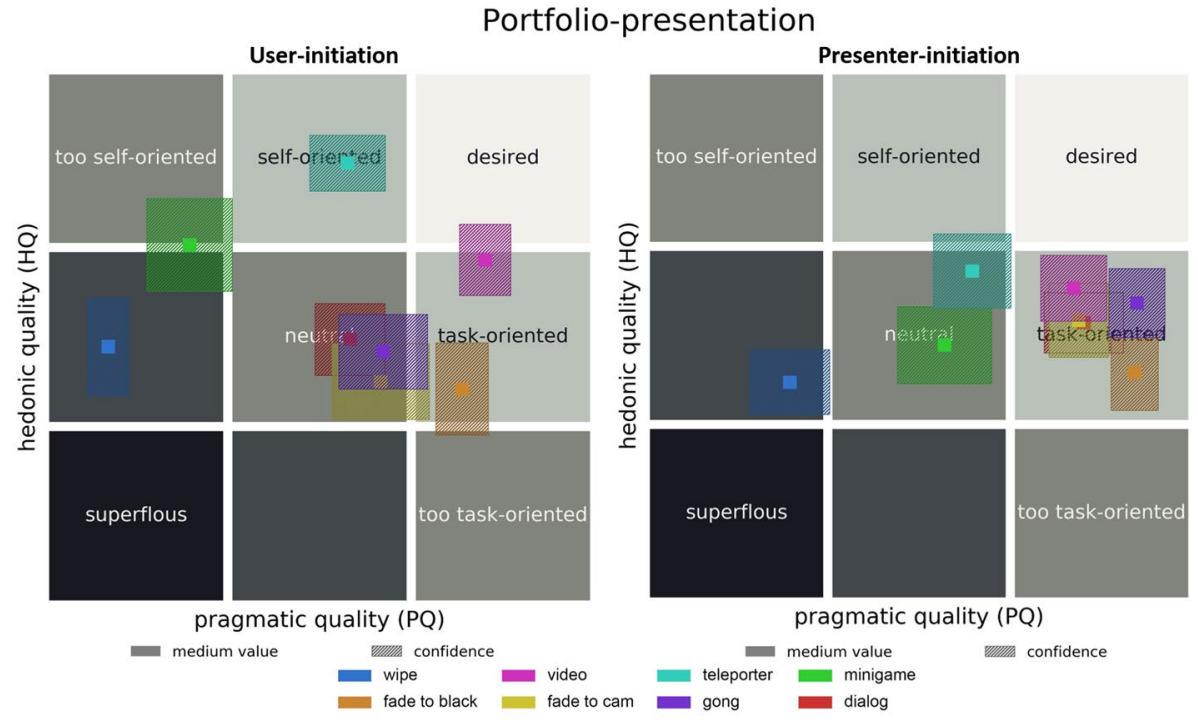

Fig. 5 AttrakDiff analysis of the portfolio presentation. It compares the eight transition techniques regarding hedonic and pragmatic aspects. Left: User-initiated techniques. Right: Presenter-initiated techniques 
is illustrated towards task-orientation. The wipe technique lie closest to the 'superfluous' field. Between the charts in Fig. 5 can a shift be observed of each technique towards higher pragmatic qualities when they were initiated by a presenter instead of the VR user. Furthermore, the techniques were rated with a similar or lower hedonic quality when a presenter initiated them compared to user-initiation.

The word-pair visualizations of both characteristics (Fig. 6) illustrate the mean scores of the single items that the AttrakDiff consists of. The above mentioned phenomena can be observed in these graphics, as well. While the user-initiated versions show a scatter among most parts of the chart, especially the items relating to the hedonic qualities are more clustered around the neutral value for presenter-initiated techniques. Only the item 'unimaginative-creative' for teleporter stands out visually with a positive value. Gong was rated notably higher at the item 'bad-good' when initiated by the presenter. The interactive techniques wipe and minigame stick out visually at most of the items in both characteristics. In contrast, minigame obtained higher pragmatic and lower hedonic scores at the presenter-initiated versions.

Figure 7 (left) shows the individual scores of the outro-transitions after the sequence assessment. Except for the interactive minigame and wipe, the techniques received similar overall scores, ranging from approx. 4 to 4.5. The first mentioned ranged techniques got a score of 1.61 and 0.72, respectively. Large visual differences between user- and presenterinitiation can be observed for dialog, teleporter, and gong in descending order. Dialog and gong received a higher score when initiated by the presenter, whereas one can observe the contrary for teleporter. The figure shows that most outro-transitions obtained a higher userinitiated score compared to their counterpart. We conducted a Mann-Whitney U test with a threshold for statistical significance of $5 \%$ on the separated data for each technique to test the differences on significance. The values for teleporter and dialog showed a statistical significant difference with $\mathrm{p}$-values of $p=0.00148$ and $p=0.00544$, respectively. Figure 7 (right) illustrates the outcome of the IPQ questionnaire. The graph shows an overall value of 4.38 for spatial presence, a value of 3.1 for involvement, 1.95 for perceived realism, and 3.14 for the overall presence. A Mann-Whitney U test with a threshold for statistical

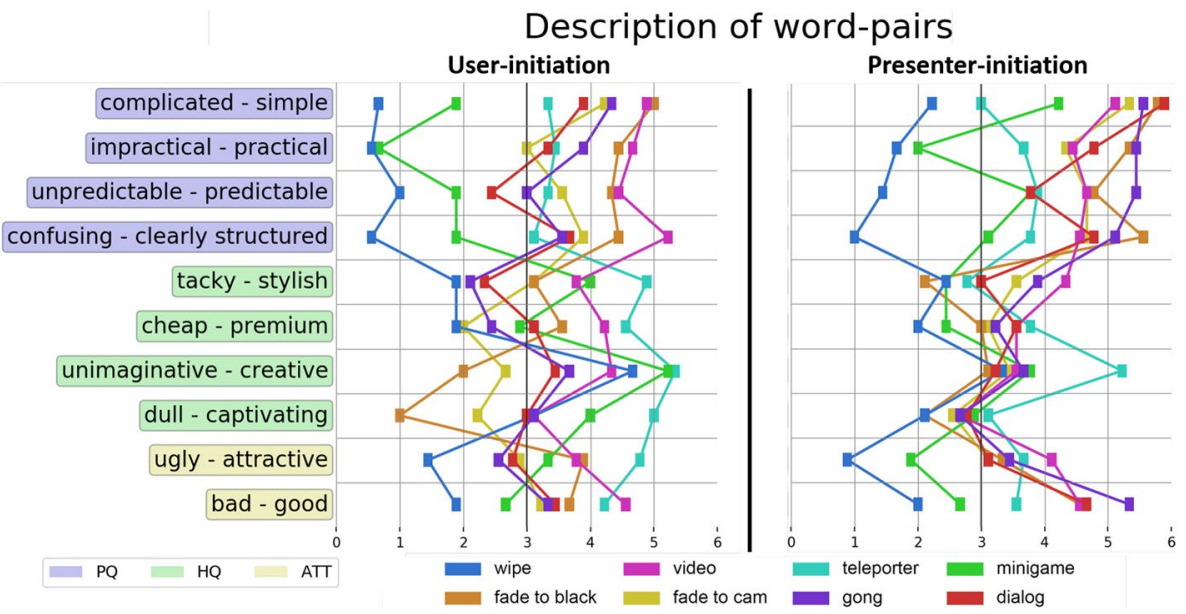

Fig. 6 AttrakDiff analysis of the description of word-pairs. It shows the mean scores of the single items that the AttrakDiff consists of. Left: User-initiated techniques. Right: Presenter-initiated techniques 

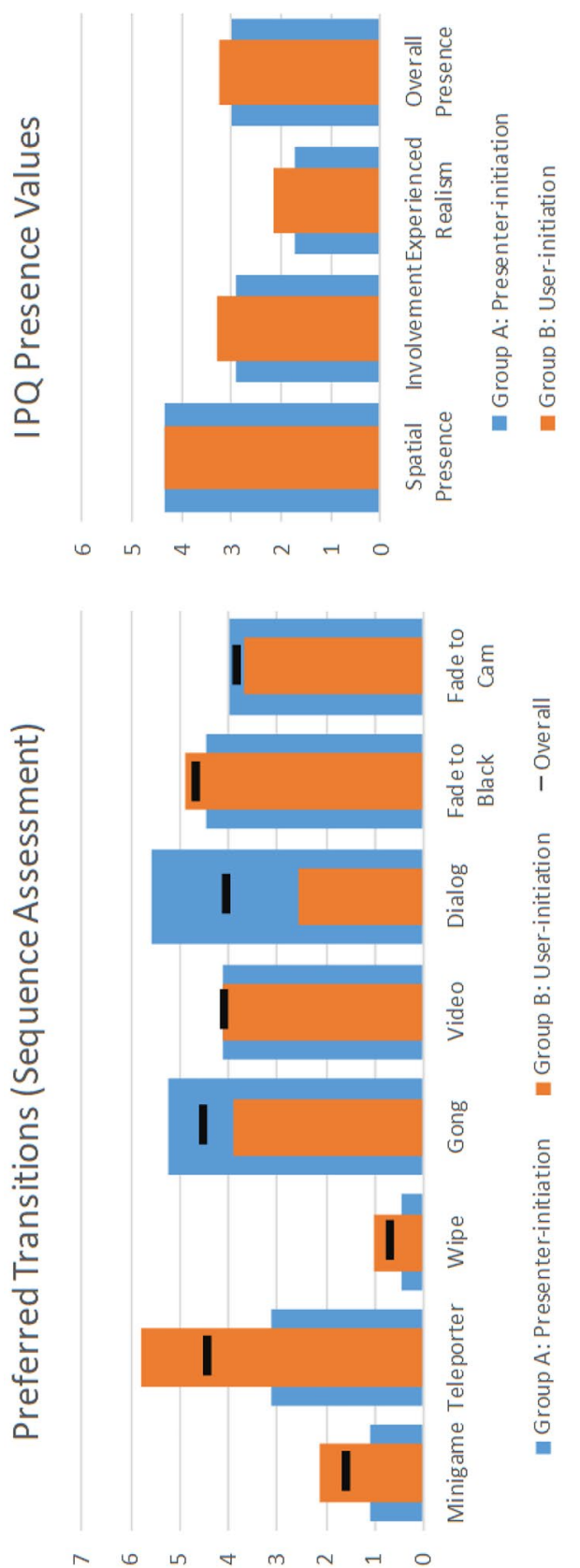

0
0
0
$\Xi$
0
0
0
0
0
0
0 惑

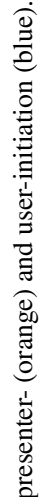
을 के

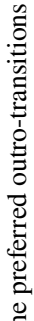
E 苛 ํํํ 
significance of $5 \%$ could not identify any significant differences between the two test conditions for any of the aspects. The user-initiated techniques' absolute values are higher for involvement, perceived realism, and overall presence (approx. 0.11 to 0.4 higher than their counterpart). The values are equal for spatial presence.

We evaluated our participants' free-text comments of by identifying different themes and aspects, utilizing affinity diagrams as proposed by Preece et al. [30]. The free text comments could be clustered into two categories. The first category consists of individual negative comments about both wipe and minigame. Participants mentioned that they would not want to complete a short game each time they exit a VR. One participant noted that, if necessary, there should be several alternative minigames to make it more diverse. Several participants mentioned that the wipe technique needs experience to accomplish the task and was complicated and not intuitive at first. They stated that 'dirt' on their glasses was connoted negatively. The second category includes several comments, which expressed that they especially liked the diversity of outro-transitions. They stated that they liked the experience of more than one transition within our study.

\subsection{Discussion of the results}

Regarding A1 (separation), our user study results indicate that our participants could separate between all considered outro-transitions and the actual content of the VR application, except for the minigame technique. Generally, our users could better distinguish between outro-transitions and the actual VR content when initiated by a presenter instead of initiated by themselves (A6). Again, participants did not immediately know that minigame was part of a transition, especially when they initiated it themselves. Gong, video, and fade to black were highest rated by our participants. For all three, there were no significant differences between the two test conditions.

There are wider variations between the techniques concerning their disturbance (A2), which impacts the overall experience of participants. Participants were overall least disturbed by the fade to black and video techniques. But the differentiated values show that gong obtained the highest score of all techniques (A6). It was recorded for the presenterinitiated condition. The evaluation particularly indicates that our participants were less disturbed when they initiated the transitions themselves, making the score of gong a phenomenon within our data. It was also the only significant different technique in this section. Except this, only both fade techniques were perceived as less disturbing when the presenter initiated them. All other techniques were rated in favor of the user-initiation.

The evaluation of how long outro-transitions may last (A3) indicates contradictions. The participants stated that dialog and fade to black felt most adequate regarding their duration after the short virtual experiences. Fade to black was the shortest outro-transition and dialog the second-longest one. A factor for this contradiction is that the participants could extend their VR experience within the dialog technique and thus could determine the end of it. Video and fade to cam were perceived as next suitable after the first-mentioned. These two were also the only techniques that were considered slightly too short and were similar short as fade to black. This insight shows a trend towards the shortest of our considered transition techniques. There were no significant differences between the test conditions regarding the duration. Whether they or the presenter initiated the transitions was not relevant for the perception of the duration for our participants (A6). 
Our participants indicated with the items from the AttrakDiff questionnaire that the two techniques teleporter and video were accepted most, regarding their hedonic and pragmatic qualities (A4). While teleporter only obtained average scores concerning A1-A3, this technique got a high score within the AttrakDiff evaluation due to its high hedonic quality. In contrast, video was rated highly due to its pragmatic quality, and it was also within the toprated techniques relating to A1-A3. Both fade techniques were only average or low rated by our participants, whereas they were favored by participants within other disciplines. Between our two test conditions, a shift took place from hedonic to pragmatic qualities when we compared user-initiation and presenter-initiation, respectively (A6). This indicates that our participants did not perceive the techniques particularly worse when a presenter took over the competence of ending the session but instead thought of this condition as more task-oriented than self-oriented as before.

Regarding the presence (A5) within the short and consecutive usage of VR, we could identify a trend similar to results in related work (e.g., [16]). Furthermore, we could not identify notable differences between the two test conditions (A6). This indicates that the competence shift, which supports the presenter roles within our scenario and enables them to end the VR session to their convenience, did not negatively impact the presence of our participants.

\section{Conclusion and future work}

In this paper, we explored transitions from a virtual world back to the physical world. We introduced this concept as outro-transitions and investigated the usage of them to enable presenters to end short and frequent VR sessions and still provide a good overall experience for VR users. We introduced a conceptual model and considered eight transition techniques than can serve as outro-transitions. This model can be used as a taxonomy to classify outro-transitions regarding three aspects that can be included in these transitions: initiation, interlude, and exit. A user study has shown that presenters can use outrotransitions to end VR sessions for VR users as intended without negative impacts on the VR users' perceived presence or overall experience. The video technique was indicated to be most suitable within our use-case, relating to its short duration, the small amount of disturbance during the usage, the separation between transition techniques and VR scene, and the acceptance of our participants. Further suitable candidates were teleporter, dialog, and gong, as these performed well in one specific aspect each. These were hedonic quality, duration and, separation/disturbance, respectively. Simple fade techniques performed well in most categories when initiated by presenters, but not best. Most notably, our participants did not favor more interactive outro-transitions such as minigame or wipe. These insights give practitioners an application-oriented advice on which techniques to use when required for presenters to end VR experiences to their convenience instead of letting VR users decide.

Future work based on our findings about outro-transitions is divided into three categories: (1) short intro-transitions, (2) longer outro-transitions, and (3) exploring the VR userpresenter relationship in asymmetric VR setups. In this work, we have lain the foundation that presenters can use outro-transitions as intended and without negative impacts. Furthermore, it can be argued that outro-transitions could be a substantial and novel part of VR offboarding, extending it by immersive aspects. This relation should also be explored in the future. But our model for transitions is not limited to be used exclusively for outro-transitions as a part of the 
offboarding process and can also be used to describe and classify other types of transition techniques. Initiation, interlude, and exit are valid concepts that can be transferred to, for example, describe intro-transitions, as exemplary shown in Table 1 . This indicates that our considered outro-transitions can be used as intro-transitions, as well. A reproduction of our study, incorporating our eight techniques as intro-transitions, could explore whether these short transition techniques can bring benefit to the users' presence, as well. If this is the case, then these short and generic transition techniques would be valuable alternatives to more elaborate techniques that require knowledge about the physical environment or include distinct authoring processes before usage. The second category for future work will explore more extensive and longer outro-transitions. Gradually transitioning from a virtual to the physical world is more applicable to wider contexts or where timekeeping is less important than the quality of the experience. In the course of this, it should also be explored in which contexts outro-transitions are generally applicable. The third direction for future work lies in the area of asymmetric VR setups, where users of the system do not exclusively use the same technology to interact with the virtual environment. In our work, the presenter user-role would use a desktop PC or laptop to get insights within the virtual world and trigger the outro-transitions. But as we consider the initiation as a separate phase within our model, this initiation is not limited for use on the same hardware that operates the VR rendering. Future work will focus on different devices for presenters and distributed VR setups. By adopting the view of presenters in our use-case, other technologies can be used in presentations or demonstrations. Outro-transitions could be triggered from mobile technologies, as well. But such an interface for presenters could also include more functionality than just starting transitions. It can also help presenters guiding VR users through virtual worlds, give presenters insights into the condition of their VR users, or letting presenters interact with the virtual content, as well. Such a tool would facilitate VR usage within presentations and help VR as a medium to be used even by non-VR-experts.

Funding Open Access funding enabled and organized by Projekt DEAL. The work is supported by the Federal Ministry of Education and Research of Germany in the project Innovative Hochschule (funding number: 03IHS071)

Open Access This article is licensed under a Creative Commons Attribution 4.0 International License, which permits use, sharing, adaptation, distribution and reproduction in any medium or format, as long as you give appropriate credit to the original author(s) and the source, provide a link to the Creative Commons licence, and indicate if changes were made. The images or other third party material in this article are included in the article's Creative Commons licence, unless indicated otherwise in a credit line to the material. If material is not included in the article's Creative Commons licence and your intended use is not permitted by statutory regulation or exceeds the permitted use, you will need to obtain permission directly from the copyright holder. To view a copy of this licence, visit http://creativecommons.org/licenses/by/4.0/.

\section{References}

1. Addelman S (1969) The generalized randomized block design. Am Stat 23(4):35-36

2. Anagram (2020) The collider. https://weareanagram.co.uk/project/the-collider. Accessed 18 Dec 2020

3. Benford S, Bowers J, Fahlén LE, Greenhalgh C (1994) Managing mutual awareness in collaborative virtual environments. In: Virtual Reality Software and Technology. World Scientific, pp. 223-236

4. Bronack S, Sanders R, Cheney A, Riedl R, Tashner J, Matzen N (2008) Presence pedagogy: Teaching and learning in a 3d virtual immersive world. Int J Learn High Educ 20(1):59-69

5. Conover WJ (1980) Practical nonparametric statistics. Wiley, New York

6. Deterding S, Dixon D, Khaled R, Nacke L (2011) From game design elements to gamefulness: defining "gamification". In: Proceedings of the 15th international academic MindTrek conference: Envisioning future media environments, pp. 9-15 
7. Deterding S, Sicart M, Nacke L, O’Hara K, Dixon D (2011) Gamification. using game-design elements in non-gaming contexts. In: CHI'11 extended abstracts on human factors in computing systems, pp. 2425-2428

8. Facebook Technologies L (2020) Oculus health and safety documents. https://www.oculus.com/legal/ health-and-safety-warnings. Accessed 19 Feb 2020

9. Flatla DR, Gutwin C, Nacke LE, Bateman S, Mandryk RL (2011) Calibration games: making calibration tasks enjoyable by adding motivating game elements. In: Proceedings of the 24th annual ACM symposium on User interface software and technology, pp. 403-412

10. Friedman M (1937) The use of ranks to avoid the assumption of normality implicit in the analysis of variance. Journal of the american statistical association 32(200):675-701

11. Hassenzahl M (2018) The thing and i: understanding the relationship between user and product. In: Funology 2. Springer, pp. 301-313

12. Hassenzahl M, Burmester M, Koller F (2003) Attrakdiff: Ein fragebogen zur messung wahrgenommener hedonischer und pragmatischer qualität. In: Mensch \& computer 2003. Springer, pp. 187-196

13. Hassenzahl M, Platz A, Burmester M, Lehner K (2000) Hedonic and ergonomic quality aspects determine a software's appeal. In: Proceedings of the SIGCHI conference on Human Factors in Computing Systems, pp. 201-208

14. Horst R, Dörner R (2018) Opportunities for virtual and mixed reality knowledge demonstration. In: 2018 IEEE International Symposium on Mixed and Augmented Reality Adjunct (ISMARAdjunct). IEEE, pp. 381-385

15. Horst R, Dörner R (2019) Integration of bite-sized virtual reality applications into pattern-based knowledge demonstration. In: 16th Workshop Virtual and Augmented Reality of the GI Group VR/ AR. Gesellschaft für Informatik, Shaker Verlag, pp. 137-148

16. Horst R, Dörner R (2019) Virtual reality forge: Pattern-oriented authoring of virtual reality nuggets. In: 25th ACM Symposium on Virtual Reality Software and Technology, pp. 1-12

17. Hovhannisyan G, Henson A, Sood S (2019) Enacting virtual reality: The philosophy and cognitive science of optimal virtual experience. In: International Conference on Human-Computer Interaction. Springer, pp. 225-255

18. HTC Corporation (2020) Htc vive cosmos product description. https://www.vive.com/de/product/ vive-cosmos/features/. Accessed 30 Jan 2020

19. HTC Corporation (2020) Htc vive product description. https://www.vive.com/. Accessed 30 Jan 2020

20. Hug T (2005) Micro learning and narration. exploring possibilities of utilization of narrations and storytelling for the designing of "micro units" and didactical micro-learning arrangements. In: fourth Media in Transition conference, vol. 6

21. Igroup Project Consortium (2020) Igroup presence questionnaire. http://www.igroup.org/pq/ipq/ index.php. Accessed 30 Jan 2020

22. Klinker G, Dutoit AH, Bauer M, Bayer J, Novak V, Matzke D (2002) Fata morgana-a presentation system for product design. In: Proceedings. International Symposium on Mixed and Augmented Reality. IEEE, pp. 76-85

23. Mann HB, Whitney DR (1947) On a test of whether one of two random variables is stochastically larger than the other. The annals of mathematical statistics. pp. 50-60

24. Mekler ED, Brühlmann F, Tuch AN, Opwis K (2017) Towards understanding the effects of individual gamification elements on intrinsic motivation and performance. Comput Hum Behav 71:525-534

25. Men L, Bryan-Kinns N, Hassard AS, Ma Z (2017) The impact of transitions on user experience in virtual reality. In: 2017 IEEE virtual reality (VR). IEEE, pp. 285-286

26. Moghadam KR, Banigan C, Ragan ED (2018) Scene transitions and teleportation in virtual reality and the implications for spatial awareness and sickness. IEEE Trans Vis Comput Graph

27. Neves Coelho D, Schmidt S, Steinicke F (2018) Kategorisierung und evaluierung von transitionen fr cave umgebungen. In: Proceedings of the GI Workshop on Virtual and Augmented Reality (GI VR/AR). Shaker Verlag

28. Norman DA (1999) Affordance, conventions, and design. Interactions 6(3), 38-43

29. Peter M, Horst R, Dörner R (2018) Vr-guide: A specific user role for asymmetric virtual reality setups in distributed virtual reality applications. In: Proceedings of the 10th Workshop Virtual and Augmented Reality of the GI Group VR/AR. Gesellschaft für Informatik, Shaker Verlag

30. Preece J, Rogers Y, Sharp H (2015) Interaction design: beyond human-computer interaction. John Wiley \& Sons

31. Schmalstieg D, Fuhrmann A, Hesina G, Szalavári Z, Encarnaçao LM, Gervautz M, Purgathofer W (2002) The studierstube augmented reality project. Presence Teleop Virt 11(1), 33-54 
32. Schubert T, Friedmann F, Regenbrecht H (2001) The experience of presence: Factor analytic insights. Presence Teleop Virt 10(3), 266-281

33. Schubert TW (2003) The sense of presence in virtual environments: A three-component scale measuring spatial presence, involvement, and realness. Zeitschrift für Medienpsychologie 15(2):69-71

34. Slater M, Steed A, McCarthy J, Marinelli F (1998) The virtual ante-room: Assessing presence through expectation and surprise

35. Slater M, Usoh M, Steed A (1994) Depth of presence in virtual environments. Presence Teleop Virt 3(2), 130-144

36. Steed A, Benford S, Dalton N, Greenhalgh C, MacColl I, Randell C, Schnädelbach H (2002) Mixedreality interfaces to immersive projection systems. In: Immersive projection technology workshop

37. Steinicke F, Bruder G, Hinrichs K, Lappe M, Ries B, Interrante V (2009) Transitional environments enhance distance perception in immersive virtual reality systems. In: Proceedings of the 6th Symposium on Applied Perception in Graphics and Visualization, pp. 19-26

38. Steinicke F, Bruder G, Hinrichs K, Steed A (2010) Gradual transitions and their effects on presence and distance estimation. Comput Graph 34(1):26-33

39. Steinicke F, Bruder G, Hinrichs K, Steed A, Gerlach AL (2009) Does a gradual transition to the virtual world increase presence? In: 2009 IEEE Virtual Reality Conference, pp. 203-210. IEEE

40. Technalysis Research; Statista estimates (2020) Average session time of virtual reality (vr) users in the united states between the 2nd and 3rd quarter of 2019, by user type. https://www.statista.com/statistics/ 1098976/average-session-time-of-vr-users-by-user-type/. Accessed 18 Dec 2020

41. Technalysis Research; Statista estimates (2020) Virtual reality and augmented reality (vr and ar) devices average session time in the united states as of 2018. https://www.statista.com/statistics/831819/ us-virtual-augmented-reality-device-average-session-time/. Accessed 17 Feb 2020

42. Technologies U (2020) Unity game engine description. https://unity.com/. Accessed 19 Feb 2020

43. User Interface Design GmbH (2020) Attrakdiff questionnaire. http://attrakdiff.de/index-en.html. Accessed 30 Jan 2020

44. Valkov D, Flagge S (2017) Smooth immersion: the benefits of making the transition to virtual environments a continuous process. In: Proceedings of the 5th Symposium on Spatial User Interaction, pp. $12-19$

45. Van Kerrebroeck H, Brengman M, Willems K (2017) When brands come to life: experimental research on the vividness effect of virtual reality in transformational marketing communications. Virtual Reality 21(4):177-191

Publisher's Note Springer Nature remains neutral with regard to jurisdictional claims in published maps and institutional affiliations.

\section{Authors and Affiliations}

\section{Robin Horst $^{1}\left(\mathbb{D} \cdot\right.$ Ramtin Naraghi-Taghi-Off $^{1} \cdot$ Linda Rau $^{1} \cdot$ Ralf Dörner $^{1}$}

Robin Horst

robin.horst@hs-rm.de

1 RheinMain University of Applied Science, Kurt-Schumacher-Ring 18, 65197 Wiesbaden, Germany 